\title{
Prediction of Sewage Wastewater Quality Based on PSO-LIBSVM
}

\author{
Qin Bin, Liu Bang
}

School of electrical and information engineering, Hunan university of technology, zhuzhou 412007, China

email: liubang1212@sina.cn

\begin{abstract}
Aiming at the problems of nonlinearity, time-varying and big lagging in an activated sludge wastewater treatment process, the forecast modeling of COD can be established according to the historical data of chemical oxygen demand(COD) collected from sewage plant, and using the LIBSVM toolbox to determine the model structure and parameters. With the use of the output error data and the particle swarm algorithm, we can optimize the parameters of support vector machine(SVM) and correct model, until the output error is minimum. The results on simulation show that the more simple modeling process, the prediction effect will be much better . Compared with the BP neural network, the standard SVM model, it can reflect the characteristics of COD distribution in the future time.
\end{abstract}

Keywords: Activated Sludge Process; LIBSVM; Particle Swarm Optimization

\section{Introduction}

The principle of SBR sewage treatment system is to use the microbial life activities such as endogenous respiration from sludge, to remove pollutants from sewage. The SBR wastewater treatment process is quite flexible, and it can be adjusted according to the situation of water quality or sewage treatment feedback, and thus provides the standard water quality standard [1]. The Sewage treatment is a highly nonlinear, strong coupling, dynamic, multiple input and multiple output process, and its process parameters change randomly. For some important 
water quality measurement index [2] (such as the concentration of chemical oxygen demand, short for COD) it lacks mature and economical online measurement instrument. Reference [3] By using the established BP neural network prediction model to predict the sewage treatment plant's output water CODcr, and the concentration of NH3-N. But its prediction accuracy depends on the network topology structure [3]. [4] By using real-time monitoring data of sewage plant, the BP and RBF neural network water COD forecast models were established respectively, But when the training samples excess, it will increases the model's learning time, otherwise, it will lower the prediction precision [4]. Due to the inherent defect of the neural network, such as easy to fall into local minimum and poor generalization ability, it caused certain constraints in practical applications.

Support Vector Machine, SVM for short, is a machine learning algorithm based on statistical learning theory. It has certain advantages over solving especially the problems of small samples, nonlinear system and overcomes the neural network's defects of poor generalization ability and easiness to fall into local optimum[5]. On the basis of existing research results, a kind of particle swarm optimization LIBSVM parameters prediction model of COD can be designed in this paper, Solving the model parameters affects the generalization ability in a certain extent. The simulation results show that the model has good forecast effect of COD and better generalization.

\section{Principle of Support Vector Machine Regression}

Describe the multivariate nonlinear regression model general form as follows: $y=f\left(x_{1}, x_{2}, \cdots, x_{L}\right)$ among them $x_{i} \in \mathfrak{R}^{n},(i=1,2, \cdots, L)$ is indicated support vector machine regression prediction model of input, $y$ is indicated the target output . The mapping function $f\left(x_{i}\right): \mathfrak{R}^{n} \rightarrow \Omega$.In the feature space by using mapping function to linear regression was carried out on the sample data. The regression estimate function is: 


$$
f(x)=\omega \varphi(x)+b
$$

Among themwas the weight vectors; $\mathrm{b}$ as bias; The coefficient $\mathrm{w}$ and $\mathrm{b}$ can be obtained by solving optimization problem. Consider fitting error, introduce the slack variable $\xi, \zeta$, The optimization problem as follows:

$$
\begin{gathered}
\min _{w, b, \xi, \zeta} \frac{1}{2}\|w\|^{2}+C \sum_{i=1}^{L}\left(\xi_{i}+\zeta_{i}\right) \\
\text { s.t }\left\{\begin{array}{c}
\left\langle w, \varphi\left(x_{i}\right)\right\rangle+b-y_{i} \leq \xi_{i}+\varepsilon \\
y_{i}-\left\langle w, \varphi\left(x_{i}\right)\right\rangle-b \leq \zeta_{i}+\varepsilon . \\
\xi_{i}, \zeta_{i} \geq 0, i=1,2, \cdots L
\end{array}\right.
\end{gathered}
$$

And then $\|w\|^{2}$ is represented as confidence limit; $\sum_{i=1}^{L}\left(\xi_{i}+\zeta_{i}\right)$ is represented the empirical risk, its function is to reduce the training error; C is for the regularization parameter controlling the degree of punishment right or wrong points sample. By formula (1) - (3) ,we can get the dual optimization expressions by formula(4):

$$
\min \frac{1}{2} \sum_{i, j=1}^{L}\left(\alpha_{i}-\alpha_{i}^{*}\right)\left(\alpha_{j}-\alpha_{j}^{*}\right) K\left(x_{i}, x_{j}\right)+\varepsilon \sum_{i=1}^{L}\left(\alpha_{i}+\alpha_{i}^{*}\right)-\sum_{i=1}^{L} y_{i}\left(\alpha_{i}-\alpha_{i}^{*}\right)
$$

Calculated by formula (4) final regression estimate function is:

$$
\hat{f}(x)=\sum_{i=1}^{L}\left(\alpha_{i}-\alpha_{i}^{*}\right) K\left(x_{i}, x\right)+\bar{b}
$$

\section{Basic Principle of PSO}

Particle Swarm Optimization is based on evolutionary group technology, the basic idea comes from the research and simulation of bird feeding behavior. 
Particle swarm optimization algorithm in essence belongs to a kind of intelligent algorithm in parallel global stochastic optimization. PSO initialized to a group of random particles, each particle has a corresponding position and velocity.Respectively $\quad X_{i d}=\left(x_{i 1}, x_{i 2}, \cdots, x_{i d}\right) \quad$ and $V_{i}=\left(v_{i 1}, v_{i 2}, \cdots, v_{i d}\right) \cdot i=1,2, \cdots L$, L stand for the population, $\mathrm{d}$ stand for the dimensions of the search space, $P_{i}=\left(p_{i 1}, p_{i 2}, \cdots, p_{i d}\right)$ stand for ith particle search to the optimal position so far; $P_{g}=\left(p_{g 1}, p_{g 2}, \cdots, p_{g d}\right)$ stand for the whole group so far to search the optimal position. Each particle has a being determined by the optimization function of adaptive value, in the process of each iteration, the velocity and position updating formula is as follows:

$$
v_{i d}^{k+1}=w * v_{i d}^{k}+c_{1} * \operatorname{rand}_{1}^{k}() *\left(p_{i d}^{k}-x_{i d}^{k}\right)+c_{2} * \operatorname{rand}_{2}^{k}() *\left(p_{g d}^{k}-x_{g d}^{k}\right)
$$

$$
x_{i d}^{k+1}=x_{i d}^{k}+v_{i d}^{k+1}
$$

In the type: $c_{1} c_{2}$ are accelerate constant; rand $_{1}$ ' rand 2 are random distribution function between $[0,1]$; In order to reduce in the process of evolution, particles leave the possibility of search space, the particle speed is usually limited to a certain range $V_{i} \in\left[-v_{\max }, v_{\max }\right] ; \mathrm{w}$ as inertia weight, played the role of trade-offs between global and local search. In order to improve the convergence speed of algorithm, we should adjust inertia weight of w[6], the formula is:

$$
w=w_{\max }-i t e r *\left(w_{\max }-w_{\min }\right) / \text { iter }_{\max }
$$

Among them, iter as the current iteration number, iter $_{\max }$ as the maximum number of iterations. 


\section{Biochemical Oxygen Demand Forecasting Model Based on PSO-LIBSVM}

According to the algorithm described above for COD prediction model, the data come form literature [7].PSO-LIBSVM parameter settings are as follows: The maximum number of iterations for 100 times ; The number of population of 20; Solution space for the two-dimensional, and the corresponding $\mathrm{C}$ and $\sigma^{2}$ values, in order to remain consistent with LIBSVM, take $g=1 / 2 \sigma^{2}$.learning factor $c 1=1.5, c 2=1.7$; Inertia coefficient $w_{\max }=0.9, w_{\min }=0.1$. The best parameters for after PSO optimization are $\mathrm{C}=18.222, g=0.01$. As the average relative absolute error and root mean square error to measure the performance of evaluation model. In order to make further comparison, using BP neural network, the standard SVM algorithm for COD modeling and simulation respectively.

The corresponding simulation results are shown in figure 1

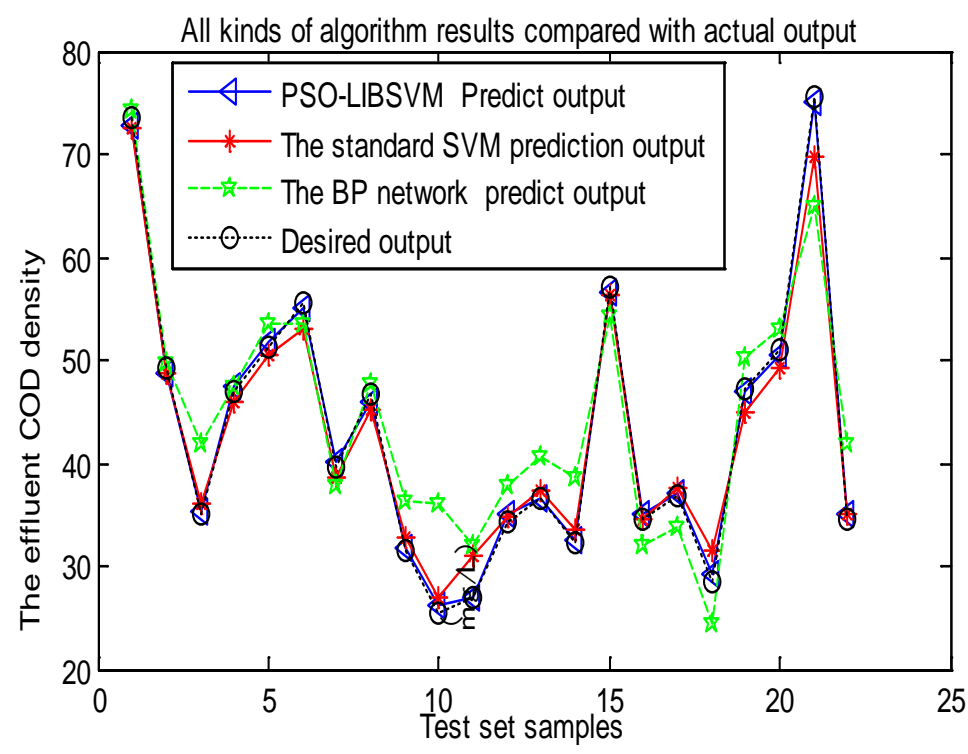

Fig. 1. Several kinds of algorithms for predict output 
Table 1 PSO - LIBSVM compared with standard SVM, BP network prediction performance

\begin{tabular}{cccc}
\hline $\begin{array}{c}\text { Indicators } \\
\text { Algorithm }\end{array}$ & $\begin{array}{c}\text { PSO- } \\
\text { LIBSVM }\end{array}$ & Standard SVM & BP neural network \\
\hline SV Number & 16 & 24 & - \\
RMSE & 0.022 & 0.033 & 0.164 \\
MRAE & 0.012 & 0.037 & 0.051 \\
\hline
\end{tabular}

Through analysis diagram 1 and table 1 , we can obtain some conclusion: In terms of fitting accuracy, PSO-LIBSVM is the highest. Root mean square error and average relative absolute value error are minimum, 0.022 and 0.012 respectively; The standard SVM is second; The BP network is the lowest. In the model generalization performance, BP show the generalization ability is poor, regression accuracy not as good as than standard SVM. Because neural network is based on empirical risk minimization. While the standard SVM performance below than PSO- LIBSVM, Because of SVM depend on the selection of its parameters to a certain extent; Through PSO optimized LIBSVM, it enhances the understanding of sparse, reduces the complexity of the model and using fewer support vectors than standard SVM.

\section{Conclusion}

This paper proposes a method of sewage water quality prediction based on PSO - LIBSVM. In view of the SVM parameters selection problem, using PSO algorithm to optimize LIBSVM penalty factor C and kernel parameters $\sigma^{2}$,and the algorithm was applied to prediction of COD. The experimental results showed that PSO - LIBSVM forecasting model not only is better generalization, higher prediction precision than standard SVM, but also has high practical value through the change trend of the predicted results can provide theory support for the sewage treatment plant operation. 


\section{Acknowledgements}

Project supported by the national natural science fund (61074067, 21106036);

Hunan Provincial Natural Science Foundation of China(13JJ3110).

\section{References}

[1] Zhang Jie, Zhang Jian-qiu, Feng Hui etc. SVM and Neural Networks Joint Approach to the Soft Measurement of COD Values in SBR Wastewater Treatment Systems[J]. Journal of Sensing Technology,2009, 22(10):15191524.

[2] Xu Fang-zhou. Design of wastewater treatment control system and research of soft sensing technique[D].Jiangnan university,2011,3.

[3] Shi Yong-jin. BP neural network model for simulation application in sewage treatment research[J].Anhui Chemical,2013,39(3):68-70.

[4] Nie Xun-ke. A Pre-treatment Model for Calculative COD of Sewage Discharge on NN[J]. Journal of Chongqing Institute of Technology .2008,22(8):156-161.

[5] W.L. WANG , M. REN. Soft-sensing Method for Wastewater Treatment Based on BP Neural Network[C].Proceedings of the 4th World Congress on Intelligent Control and Automation, Shanghai, P.R. China,2002:2330-2332.

[6] MELGANI.F, YAKOUB B. Classification of Electrocardiogram Signals With Support Vector Machines and Particle Swarm Optimization[J].IEEE Transactions on Information Technology in Biomedicine.2008:667-677.

[7] Luo Teng-fei. Predictive Indicators of Water in Wastewater Treatment Based on Improved BP Artificial Neural Network[D].Inner Mongolia agricultural university,2012,5. 